\title{
Cognitive Transmissions with Multiple Relays in Cognitive Radio Networks
}

\author{
Yulong Zou, Student Member, IEEE, Yu-Dong Yao, Senior Member, IEEE, and Baoyu Zheng, Member, IEEE
}

\begin{abstract}
In cognitive radio networks, each cognitive transmission process typically requires two phases: the spectrum sensing phase and data transmission phase. In this paper, we investigate cognitive transmissions with multiple relays by jointly considering the two phases over Rayleigh fading channels. We study a selective fusion spectrum sensing and best relay data transmission (SFSS-BRDT) scheme in multiple-relay cognitive radio networks. Specifically, in the spectrum sensing phase, only the initial spectrum sensing results, which are received from the cognitive relays and decoded correctly at a cognitive source, are selected and used for fusion. In the data transmission phase, only the best relay is utilized to assist the cognitive source for data transmissions. Under the constraint of satisfying a required probability of false alarm of spectrum holes (for the protection of the primary user), we derive an exact closed-form expression of the spectrum hole utilization efficiency for the SFSS-BRDT scheme, which is used as a measure to quantify the percentage of spectrum holes utilized by the cognitive source for its successful data transmissions. For the comparison purpose, we also examine the spectrum hole utilization efficiency for a fixed fusion spectrum sensing and best relay data transmission (FFSS-BRDT) scheme, where all the initial spectrum sensing results are used for fusion without any refined selection. Numerical results show that, under a target probability of false alarm of spectrum holes, the SFSSBRDT scheme outperforms the FFSS-BRDT scheme in terms of the spectrum hole utilization efficiency. Moreover, the spectrum hole utilization efficiency of the SFSS-BRDT scheme always improves as the number of cognitive relays increases, whereas the FFSS-BRDT scheme's performance improves initially and degrades eventually after a critical number of cognitive relays. It is also shown that a maximum spectrum hole utilization efficiency can be achieved through an optimal allocation of the time durations between the spectrum sensing and data transmission phases for both the FFSS-BRDT and SFSS-BRDT schemes.
\end{abstract}

Index Terms-Cognitive transmissions, cognitive radio, multiple relays, spectrum sensing, best relay selection, spectrum hole utilization efficiency.

Manuscript received May 16, 2010; revised August 4, 2010 and September 21, 2010; accepted November 15, 2010. The associate editor coordinating the review of this paper and approving it for publication was D. Hong.

Y. Zou is with the Institute of Signal Processing and Transmission, Nanjing University of Posts and Telecommunications, Nanjing, Jiangsu 210003, China, and with the Electrical and Computer Engineering Department, Stevens Institute of Technology, Hoboken, NJ 07030, USA (e-mail: zouyulong198412@126.com, yzou1@stevens.edu).

Y.-D. Yao is with the Electrical and Computer Engineering Department, Stevens Institute of Technology, Hoboken, NJ 07030, USA (e-mail: yyao@stevens.edu).

B. Zheng is with the Institute of Signal Processing and Transmission, Nanjing University of Posts and Telecommunications, Nanjing, Jiangsu 210003, China (e-mail: zby@njupt.edu.cn).

This work was partially supported by the Postgraduate Innovation Program of Scientific Research of Jiangsu Province (Grant Nos. CX08B_080Z, CX09B_150Z) and the National Natural Science Foundation of China (Grant No. 60972039).

Digital Object Identifier 10.1109/TWC.2010.120610.100830

\section{INTRODUCTION}

$\mathbf{C}$ OGNITIVE radio is emerging as a promising technology that enables unlicensed users, also referred to as cognitive users (or secondary users), to communicate with each other over licensed bands through detecting spectrum holes [1]-[3]. As discussed in [4] and [5], each cognitive transmission process typically consists of two essential phases: 1) a spectrum sensing phase, in which a cognitive source attempts to detect an available spectrum hole; and 2) a data transmission phase, in which the secondary data traffic is transmitted to a destination through the detected spectrum hole. The two individual phases have been studied extensively in terms of different sensing [6]-[11] or different transmission [12]-[21] techniques.

However, the two individual phases can not be designed and optimized separately, since they affect each other [5]. To be specific, when an available spectrum hole is not perceived by the cognitive source during a certain time duration, the spectrum hole utilization efficiency will be impaired. We may increase the time duration of spectrum sensing phase to alleviate the problem of misdetection of spectrum holes, which, however, comes at the expense of a transmission performance reduction since less time is now available for the data transmission phase [5]. The work of [4] investigated the sensing-andthroughput tradeoff in terms of the maximization of secondary throughput with a primary user protection constraint, where a closed-form secondary throughput expression is derived over additive white Gaussian noise (AWGN) channels. In [5], we have explored the sensing-and-transmission tradeoff in two cognitive radio network scenarios (i.e., cognitive transmissions without relay and with single-relay), where closed-form outage probability expressions with a primary user protection constraint are derived for the two scenarios considering Rayleigh fading environments. Notice that the cognitive transmissions with multiple relays are yet to be investigated in cognitive radio networks.

In traditional (non-cognitive radio) multiple-relay networks, three relay protocols (i.e., fixed relaying, selection relaying and incremental relaying) have been studied extensively in [14]. The advantages of such relaying protocols are achieved at the cost of a reduction in spectral efficiency, since the relays used transmit on orthogonal channels to avoid interfering each other. To address the shortcoming of an inefficient utilization of the spectrum resource, a best-relay selection protocol has been investigated in [19]-[21], where only the "best" relay is selected to forward a source node's signal and thus only two channels (i.e., the best relay link and direct link) are required regardless of the number of relays. It has been shown in [19] 
that the best-relay selection scheme can achieve the same diversity-multiplexing tradeoff performance as the traditional protocols where all relays are involved in forwarding the source node's signal. Accordingly, the best-relay selection is also an attractive relay protocol for cognitive radio networks due to its spectrum efficiency. However, compared with the best-relay transmission in traditional wireless networks, cognitive radio networks face an additional challenging issue, i.e., mutual interference between the primary and the cognitive users, especially in a relay network scenario.

In addition, the existing cooperative spectrum sensing works [9], [11] assume the perfect transmission of initial spectrum sensing results to a fusion center over a dedicated channel (also called common control channel). Although the initial sensing results consist of only a few information bits, the cognitive users should scan the licensed channel periodically (e.g., in millisecond scale) [2], which results in a non-negligible rate of the initial sensing result transmission. Moreover, the common control channel resources are typically limited and wireless fading should be considered, thus the assumption of perfect transmission of initial sensing results is not valid in practical cognitive radio systems.

In this paper, we investigate the cognitive transmissions with multiple relays by jointly considering the spectrum sensing and data transmissions phases over Rayleigh fading channels. The main contributions of this paper are described as follows. First, we study a selective fusion spectrum sensing and best relay data transmission (SFSS-BRDT) scheme, where only the initial spectrum sensing results (from the multiple cognitive relays) received and decoded successfully at the cognitive source are used for fusion and then only the best relay is selected to assist the cognitive source for its data transmissions. Second, an exact closed-form expression of the spectrum hole utilization efficiency, which is used as a measure to quantify the percentage of spectrum holes utilized, is derived for the SFSS-BRDT scheme to evaluate its performance. Third, for the purpose of performance comparison, we further investigate a fixed fusion spectrum sensing and best relay data transmission (FFSS-BRDT) scheme, where the difference from the SFSS-BRDT scheme lies that all initial sensing results from the cognitive relays are used for fusion without any refined selection. Finally, we also derive a closed-form expression of the spectrum hole utilization efficiency for the FFSS-BRDT scheme over Rayleigh fading channels.

The remainder of this paper is organized as follows. In Section II, we describe the system model of cognitive transmissions and present the signal modeling for the SFSS-BRDT as well as the FFSS-BRDT schemes. Section III derives closedform expressions of the spectrum hole utilization efficiency for the SFSS-BRDT and FFSS-BRDT schemes over Rayleigh fading channels. Next, in Section IV, we conduct the numerical evaluations and computer simulations for the spectrum hole utilization efficiency of the SFSS-BRDT and FFSS-BRDT schemes, showing the advantage of the former scheme. Finally, we provide concluding remarks in Section V. The notations used throughout this paper are illustrated in Table 1.

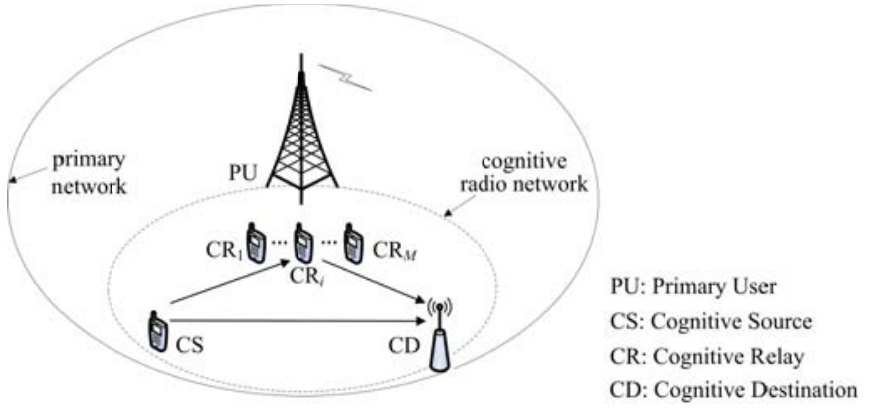

Fig. 1. Coexistence of a primary network and a cognitive radio network.

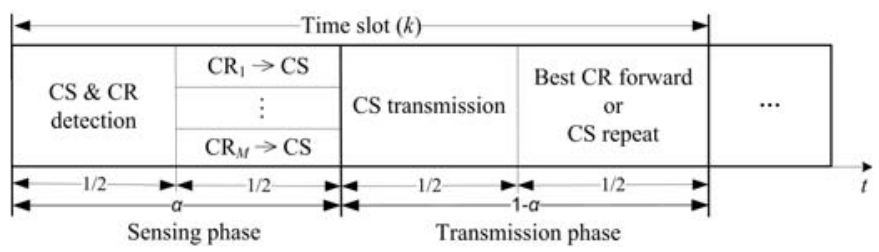

Fig. 2. Time slotted structure of the cognitive transmissions with multiple relays.

\section{Cognitive Transmission Protocols in Multiple-Relay Cognitive Radio Networks}

\section{A. System Description}

As shown in Fig. 1, we consider a cognitive radio network, where multiple cognitive relays (CRs) are available to assist a cognitive source (CS) for both the spectrum sensing and data transmission phases, i.e., CRs first help CS detect a spectrum hole to find a transmission opportunity and then assist CS for its data transmissions to a cognitive destination (CD). Following [14], [16] and considering for practicability, a halfduplex relaying mode is adopted for CRs. Notice that there are $M$ CRs as denoted by $\mathcal{R}=\left\{\mathrm{CR}_{i} \mid i=1,2, \cdots, M\right\}$. Fig. 2 illustrates a time slotted structure of the cognitive transmissions with multiple relays. In order to avoid interfering the primary users, we assume that there is a common control channel when CRs forward their initial spectrum sensing results to CS [11]. As seen from Fig. 2, each cognitive transmission process includes two phases (i.e., the spectrum sensing and data transmission phases), where the parameter $\alpha$ is referred to as spectrum sensing overhead, which can be adjusted to optimize the performance of cognitive transmissions.

Fig. 2 shows that the spectrum sensing phase consists of two sub-phases. In the first sub-phase, CS and CRs independently detect whether or not there is a spectrum hole. Then, in the subsequent sub-phase, all CRs forward their detection results (also referred to as initial spectrum sensing results) to CS over $M$ orthogonal common control sub-channels. Here, we consider two fusion strategies for the spectrum sensing: selective fusion and fixed fusion. In the selective fusion spectrum sensing (SFSS) scheme, a forward error detection code (such as cyclic redundancy check) is utilized by CRs to encode their initial spectrum sensing results, in addition to a forward error correction code, e.g., Turbo code. The encoded bits are transmitted to CS which will decode the received signals and combines the successfully decoded outcomes only, i.e., only the successfully decoded outcomes are selected and 
TABLE I

LIST OF THE NOTATIONS USED THROUGHOUT THIS PAPER.

\begin{tabular}{|c|c|}
\hline$B T, B_{c} T_{c}$ & $\begin{array}{l}\text { The time-bandwidth products of the licensed primary channel and the common control } \\
\text { channel, respectively. }\end{array}$ \\
\hline$C_{m}$ & $\begin{array}{l}\text { A non-empty set consisting of these cognitive relays whose initial spectrum sensing results } \\
\text { are received and decoded successfully at a cognitive source. }\end{array}$ \\
\hline$D_{m}$ & $\begin{array}{l}\text { A non-empty set consisting of these cognitive relays that are able to decode the signal } \\
\text { transmitted from the cognitive source. }\end{array}$ \\
\hline$H_{p}(k)$ & The status of the licensed primary channel at time slot $k$. \\
\hline$H_{0}, H_{1}$ & $\begin{array}{l}\text { Events representing the licensed channel being unoccupied and occupied by a primary user, } \\
\text { respectively. }\end{array}$ \\
\hline $\begin{array}{l}\hat{H}_{s}(k, 1) \\
\hat{H}_{i}(k, 1)\end{array}$ & $\begin{array}{l}\text { The initial spectrum sensing results detected by the cognitive source and } i \text {-th cognitive relay, } \\
\text { respectively, at time slot } k \text {. }\end{array}$ \\
\hline$\hat{H}_{s}^{i}(k, 1)$ & $\begin{array}{l}\text { The decoded outcome of an initial spectrum sensing result received at the cognitive source } \\
\text { from the } i \text {-th cognitive relay. }\end{array}$ \\
\hline $\mathrm{Pd}_{i, 1}, \mathrm{Pf}_{i, 1}$ & $\begin{array}{l}\text { The individual probabilities of detection and false alarm of spectrum holes at the } i \text {-th } \\
\text { cognitive relay, respectively. }\end{array}$ \\
\hline $\mathrm{Pd}_{s, 1}, \mathrm{Pf}_{s, 1}$ & $\begin{array}{l}\text { The individual probabilities of detection and false alarm of spectrum holes at the cognitive } \\
\text { source, respectively. }\end{array}$ \\
\hline $\mathrm{Pd}_{s}, \mathrm{Pf}_{s}$ & $\begin{array}{l}\text { The overall probabilities of detection and false alarm of spectrum holes at the cognitive } \\
\text { source after fusion, respectively. }\end{array}$ \\
\hline$P_{s}, P_{p}$ & The transmit powers at a secondary user and a primary user, respectively. \\
\hline$\gamma_{s}, \gamma_{p}$ & The transmit signal-to-noise ratios (SNR) at a secondary user and a primary user, respectively. \\
\hline
\end{tabular}

used for fusion. However, in the fixed fusion spectrum sensing (FFSS) scheme, only a forward error correction code is used by CRs to encode their initial sensing results, and CS decodes the received signals from CRs and combines all the decoded outcomes based on a given fusion rule. Presently, there are several choices of fusion rule available, such as "AND", "OR" , and majority rule in the literature [9], [11]. Note that an "AND" fusion rule is used throughout this paper without the consideration of other rules.

In the data transmission phase, we consider the use of a best relay data transmission (BRDT) strategy. As shown in Fig. 2, there are also two sub-phases for the data transmissions. If a spectrum hole was detected earlier (in the sensing phase), CS will start transmitting its data to CD and CRs in the first data transmission sub-phase. Then, all CRs attempt to decode the CS's signal and those CRs which decode successfully constitute a set $D$, called a decoding set. Notice that we can employ a cyclic redundancy check code (CRC) to determine whether a CR decodes its received signal successfully or not. Specifically, if the CRC checking passes at the CR, it is viewed as a successfully decoded relay and is added to the decoding set. Accordingly, the sample space of all the possible decoding sets is described as $\left\{D \in \emptyset \cup D_{m}, m=1,2, \cdots 2^{M}-1\right\}$, where $\cup$ represents set union, $\emptyset$ is the empty set, and $D_{m}$ is a non-empty subcollection of the $M$ cognitive relays. In the second data transmission sub-phase, if the decoding set $(D)$ is not empty, the best relay (i.e., with the highest instantaneous signal-to-interference-plus-noise ratio) chosen within the decoding set will forward its decoded result to CD. If $D$ is empty, i.e., no relay is able to decode the CS's signal successfully, CS will repeat the transmission of the original signal to $\mathrm{CD}$ through its direct link. Finally, CD combines the two copies of the received signals using the maximum ratio combining (MRC) method, and gives an estimation of the original signal. The reason for choosing MRC is that it can achieve a better performance compared with the other two combining methods, i.e., the selective diversity combining (SDC) and the equal gain combining (EGC).

It is noted that, although the scenario considered is with the single cognitive source-destination pair and somewhat specific, it can be applied and extended to a more generic scenario with multiple cognitive source-destination pairs by designing an additional multiple access protocol. To be specific, if a random access strategy is considered, we can allow multiple cognitive sources to independently sense the licensed channel with the assistance of multiple relays. Then, the source which first detects a spectrum hole starts its data transmissions by using the best relay selection scheme. On the other hand, if a static multiple access strategy is considered, the cognitive sources can cooperatively sense the licensed primary channel and then access the detected spectrum hole through a roundrobin scheduling (or, an orthogonal channelization method). In addition, for the scenario consisting of multiple licensed primary channels, a cognitive source is able to sense the multiple channels simultaneously by using filter banks [22]. Once one (or more than one) spectrum hole was found, the cognitive source starts its data transmissions over the detected spectrum hole.

Each wireless link between any two nodes as depicted in Fig. 1 is modeled as a Rayleigh fading channel and the fading process is considered as constant during one time slot. The additive white Gaussian noise (AWGN) at all receivers is 
modeled as a complex Gaussian random variable (RV) with zero mean and variance $N_{0}$. For notational convenience, let $H_{p}(k)$ represent, for time slot $k$, whether or not there is a spectrum hole. Specifically, $H_{p}(k)=H_{0}$ represents that a spectrum hole is available, i.e., the channel is unoccupied by the primary user (PU); otherwise, $H_{p}(k)=H_{1}$. We model $H_{p}(k)$ as a Bernoulli random variable with parameter $\mathrm{P}_{\mathrm{a}}$ (the probability of the channel being available for secondary users), i.e., $\operatorname{Pr}\left(H_{p}(k)=H_{0}\right)=\mathrm{P}_{\mathrm{a}}$ and $\operatorname{Pr}\left(H_{p}(k)=H_{1}\right)=1-\mathrm{P}_{\mathrm{a}}$.

\section{B. Signal Modeling}

1) Modeling of the SFSS-BRDT scheme: In the following, we first formulate the signal model of the SFSS-BRDT scheme, where the SFSS and BRDT strategies are used for the spectrum sensing and data transmission phases, respectively. In the first sub-phase of time slot $k$, the signal received at CS is expressed as

$$
y_{s}(k, 1)=\sqrt{P_{p}} h_{p s}(k) \theta(k, 1)+n_{s}(k, 1)
$$

where $h_{p s}(k)$ is the fading coefficient of the channel from PU to $\mathrm{CS}, n_{s}(k, 1)$ is AWGN with zero mean and variance $N_{0}$, and $\theta(k, 1)$ is defined as

$$
\theta(k, 1)= \begin{cases}0, & H_{p}(k)=H_{0} \\ x_{p}(k, 1), & H_{p}(k)=H_{1}\end{cases}
$$

where $x_{p}(k, 1)$ is the transmit signal of PU in the first subphase of time slot $k$. Notice that $H_{p}(k)=H_{0}$ denotes that the channel is unoccupied by PU and nothing is transmitted from PU, and $H_{p}(k)=H_{1}$ represents that a PU signal is transmitted. Meanwhile, the signals received at all CRs can be written as

$$
y_{i}(k, 1)=\sqrt{P_{p}} h_{p i}(k) \theta(k, 1)+n_{i}(k, 1), \quad i \in \mathcal{R}
$$

where $h_{p i}(k)$ is the fading coefficient of the channel from PU to $\mathrm{CR}_{i}$ and $n_{i}(k, 1)$ is $\mathrm{AWGN}$ with zero mean and variance $N_{0}$. Based on the received signals as given by Eq. (1) and Eq. (2), CS and CRs obtain their initial spectrum sensing results (as denoted by $\hat{H}_{s}(k, 1)$ and $\hat{H}_{i}(k, 1)$, respectively) by using a given detection approach. At present, we have many choices of detector available, such as energy detector [6], matched filter detector [3], and cyclostationary feature detector [7], [8]. In this paper, we use the energy detector [6], [10] to evaluate the spectrum sensing performance. The reasons for choosing the energy detector are twofold: (1) We want to show the effect of the use of multiple relays for cognitive transmissions. Hence, the choice of detector is not critical; (2) We model the signal as a random variable with known variance, and thus the energy detector is optimal [10].

In the subsequent sub-phase, all CRs encode their initial results by using a concatenated forward error detection and correction code (e.g., CRC-Turbo), giving an encoded bit stream that is mapped into a constellation symbol as denoted by $x_{i}(k, 2)$ by using a certain modulation scheme (e.g., BPSK and QAM). Then, CRs transmit the modulated symbols to CS over $M$ orthogonal common control sub-channels. Hence, the received signals at CS from CRs can be expressed as

$$
y_{s}^{i}(k, 2)=\sqrt{P_{s}} h_{i s}(k) x_{i}(k, 2)+n_{s}^{i}(k, 2), \quad i \in \mathcal{R}
$$

where $h_{i s}(k)$ is the fading coefficient of the channel from $\mathrm{CR}_{i}$ to $\mathrm{CS}$, and $n_{s}^{i}(k, 2)$ is AWGN with zero mean and variance $N_{0}$. From Eq. (3), CS attempts to decode $\hat{H}_{i}(k, 1)$ from its received signal and the decoded outcome (after Turbo decoding and CRC checking) is denoted by $\hat{H}_{s}^{i}(k, 2)$. Note that, according to the coding theorem, if a channel outage event occurs, i.e., channel capacity is smaller than data rate, the receiver is deemed to fail to decode and the CRC check is assumed to fail to pass. For notational convenience, let a set $C$ represent the CRs whose initial sensing results are received and decoded successfully at CS. Accordingly, the sample space of such a set is given by $\left\{C \in \emptyset \cup C_{m}, m=\right.$ $\left.1,2, \cdots, 2^{M}-1\right\}$, where $C_{m}$ is a non-empty subcollection of the $M$ cognitive relays.

- Case $C=\emptyset$ : This case corresponds to that CS fails to

decode any of the initial spectrum sensing results from CRs. Note that an initial sensing result $\hat{H}_{i}(k, 1)$ carries 1-bit information only in an information-theoretic sense and the time-bandwidth product of the common control channel is $B_{c} T_{c}$. Hence, the transmission rate of such an initial sensing result over the common control channel is given by $1 /\left(B_{c} T_{c}\right)$. Accordingly, the case $C=\emptyset$ can be described from Eq. (3) as

$$
\frac{\alpha}{2 M} \log _{2}\left(1+\left|h_{i s}(k)\right|^{2} \gamma_{s}\right)<\frac{1}{B_{c} T_{c}}, \quad i \in \mathcal{R}
$$

where $\gamma_{s}=P_{s} / N_{0}$, and the factor $1 / M$ is due to the fact that each CR is assigned with $1 / M$ fraction of the common control channel. Therefore, given that case $C=\emptyset$ has occurred, the final spectrum sensing result fused at CS, $\hat{H}_{s}(k)$, is expressed as

$$
\hat{H}_{s}(k \mid C=\emptyset)=\hat{H}_{s}(k, 1)
$$

which implies that none of the CRs' initial spectrum sensing results is utilized for fusion at CS.

- Case $C=C_{m}$ : CS successfully decodes these initial spectrum sensing results from the $\mathrm{CRs}$ in set $C$, i.e.,

$$
\begin{array}{ll}
\frac{\alpha}{2 M} \log _{2}\left(1+\left|h_{i s}(k)\right|^{2} \gamma_{s}\right)>\frac{1}{B_{c} T_{c}}, & i \in C_{m} \\
\frac{\alpha}{2 M} \log _{2}\left(1+\left|h_{j s}(k)\right|^{2} \gamma_{s}\right)<\frac{1}{B_{c} T_{c}}, & j \in \bar{C}_{m}
\end{array}
$$

where $\bar{C}_{m}=\mathcal{R}-C_{m}$ is the complementary set of $C_{m}$. In the given case $C=C_{m}$, CS combines $\hat{H}_{s}(k, 1)$ and $\hat{H}_{s}^{i}(k, 2)$ through a given fusion rule, leading to its final decision. Since we just want to show the sensing-transmission tradeoff in a multiple-relay cognitive radio network, the choice of fusion rule is not critical and an "AND" fusion is used throughout this paper without the consideration of other rules, e.g., "OR" and majority rule. Thus, the final sensing result $\hat{H}_{s}(k)$ is expressed as

$$
\hat{H}_{s}\left(k \mid C=C_{m}\right)=\hat{H}_{s}(k, 1) \underset{i \in C_{m}}{\otimes} \hat{H}_{i}(k, 1)
$$

which means that $\hat{H}_{s}\left(k \mid C=C_{m}\right)=H_{0}$ (i.e., a spectrum hole is detected) if all initial spectrum sensing results imply a spectrum hole available, and $\hat{H}_{s}\left(k \mid C_{m}\right)=H_{1}$ otherwise. Next, we develop signal modeling for the data transmission phase of the SFSS-BRDT scheme. In the first part of the data 
transmission, i.e., the third sub-phase of time slot $k$, the signal received at $\mathrm{CD}$ is expressed as

$y_{d}(k, 3)=h_{s d}(k) \sqrt{P_{s}} \beta(k, 3)+h_{p d}(k) \sqrt{P_{p}} \theta(k, 3)+n_{d}(k, 3)$

where $h_{s d}(k)$ and $h_{p d}(k)$ are the fading coefficients of the channel from CS to CD and that from PU to CD, respectively, and the parameters $\beta(k, 3)$ and $\theta(k, 3)$ are defined as

$$
\beta(k, 3)= \begin{cases}x_{s}(k), & \hat{H}_{s}(k)=H_{0} \\ 0, & \hat{H}_{s}(k)=H_{1}\end{cases}
$$

and

$$
\theta(k, 3)= \begin{cases}0, & H_{p}(k)=H_{0} \\ x_{p}(k, 3), & H_{p}(k)=H_{1}\end{cases}
$$

where $x_{s}(k)$ and $x_{p}(k, 3)$ are the transmit signals of $\mathrm{CS}$ and PU, respectively. Meanwhile, the signals received at CRs can be written as

$y_{i}(k, 3)=h_{s i}(k) \sqrt{P_{s}} \beta(k, 3)+h_{p i}(k) \sqrt{P_{p}} \theta(k, 3)+n_{i}(k, 3)$

where $h_{s i}(k)$ and $h_{p i}(k)$ are the fading coefficients of the channel from CS to $\mathrm{CR}_{i}$ and that from $\mathrm{PU}$ to $\mathrm{CR}_{i}$, respectively. In the fourth sub-phase, there are two possible cases for the data transmission depending on whether or not the decoding set $(D)$ is empty. Let $D=\emptyset$ represent the first case of an empty decoding set and $D=D_{m}$ correspond to the other case, where $D_{m}$ is a non-empty subcollection set of all cognitive relays (CRs).

- Case $D=\emptyset$ : This case corresponds to the scenario where all CRs fail to decode CS's signal from Eq. (9), implying

$$
\frac{(1-\alpha)}{2} \log _{2}\left(1+\frac{\left|h_{s i}(k)\right|^{2} \gamma_{s}|\beta(k, 3)|^{2}}{\left|h_{p i}(k)\right|^{2} \gamma_{p}|\theta(k, 3)|^{2}+1}\right)<R, i \in \mathcal{R}
$$

where $R$ is the data rate of CS, $\mathcal{R}$ represents a set consisting of all $M$ CRs, $\gamma_{s}=P_{s} / N_{0}$, and $\gamma_{p}=P_{p} / N_{0}$. In the given case $D=\emptyset$, CS will determine whether or not to repeat the transmission of signal $x_{s}(k)$ to $\mathrm{CD}$ depending on its final decision $\hat{H}_{s}(k)$ obtained during the spectrum sensing phase, and thus the received signal at $\mathrm{CD}$ is given by

$$
\begin{aligned}
y_{d}(k, 4 \mid D=\emptyset)= & h_{s d}(k) \sqrt{P_{s}} \beta(k, 4)+h_{p d}(k) \sqrt{P_{p}} \theta(k, 4) \\
& +n_{d}(k, 4)
\end{aligned}
$$

where

$$
\beta(k, 4)= \begin{cases}x_{s}(k), & \hat{H}_{s}(k)=H_{0} \\ 0, & \hat{H}_{s}(k)=H_{1}\end{cases}
$$

and

$$
\theta(k, 4)= \begin{cases}0, & H_{p}(k)=H_{0} \\ x_{p}(k, 4), & H_{p}(k)=H_{1}\end{cases}
$$

By combining Eq. (8) and Eq. (11) with the maximum ratio combining (MRC) method, CD can achieve an enhanced signal version with a signal-to-interference-and-noise ratio (SINR) as given by

$$
\begin{aligned}
& \operatorname{SINR}_{\mathbf{d}}(D=\emptyset) \\
& =\frac{\left|h_{s d}(k)\right|^{2} \gamma_{s}|\beta(k, 3)|^{2}+\left|h_{s d}(k)\right|^{2} \gamma_{s}|\beta(k, 4)|^{2}}{\left|h_{p d}(k)\right|^{2} \gamma_{p}|\theta(k, 3)|^{2}+\left|h_{p d}(k)\right|^{2} \gamma_{p}|\theta(k, 4)|^{2}+2}
\end{aligned}
$$

- Case $D=D_{m}$ : This case corresponds to the scenario where CRs in decoding set $D_{m}$ are able to decode CS's signal successfully, i.e.,

$$
\begin{aligned}
& \frac{(1-\alpha)}{2} \log _{2}\left(1+\frac{\left|h_{s i}(k)\right|^{2} \gamma_{s}|\beta(k, 3)|^{2}}{\left|h_{p i}(k)\right|^{2} \gamma_{p}|\theta(k, 3)|^{2}+1}\right)>R, \quad i \in D_{m} \\
& \frac{(1-\alpha)}{2} \log _{2}\left(1+\frac{\left|h_{s j}(k)\right|^{2} \gamma_{s}|\beta(k, 3)|^{2}}{\left|h_{p j}(k)\right|^{2} \gamma_{p}|\theta(k, 3)|^{2}+1}\right)<R, \quad j \in \bar{D}_{m}
\end{aligned}
$$

where $\bar{D}_{m}=\mathcal{R}-D_{m}$ is the complementary set of $D_{m}$. Without loss of generality, consider that $\mathrm{CR}_{i}$ is selected to forward its correctly decoded result. Hence, given that case $D=D_{m}$ occurred and $\mathrm{CR}_{i}$ is selected, the signal received at $\mathrm{CD}$ in the fourth sub-phase is written as

$$
\begin{aligned}
y_{d}\left(k, 4 \mid D=D_{m}\right)= & h_{i d}(k) \sqrt{P_{s}} x_{s}(k)+h_{p d}(k) \sqrt{P_{p}} \theta(k, 4) \\
& +n_{d}(k, 4)
\end{aligned}
$$

where $h_{i d}(k)$ is the fading coefficient of the channel from $\mathrm{CR}_{i}$ to CD. Combining Eq. (8) and Eq. (14) with MRC, the corresponding received SINR is given by

$$
\begin{aligned}
& \operatorname{SINR}_{\mathrm{d}}\left(D=D_{m}, \mathrm{CR}_{i}\right) \\
& =\frac{\left|h_{s d}(k)\right|^{2} \gamma_{s}+\left|h_{i d}(k)\right|^{2} \gamma_{s}}{\left|h_{p d}(k)\right|^{2} \gamma_{p}|\theta(k, 3)|^{2}+\left|h_{p d}(k)\right|^{2} \gamma_{p}|\theta(k, 4)|^{2}+2}
\end{aligned}
$$

In obtaining Eq. (15), we have used $\beta(k, 3)=x_{s}(k)$ in the given case $D=D_{m}$. In general, the relay, which successfully decodes CS's signal and achieves the highest received SINR at $\mathrm{CD}$, is viewed as the "best" one. As a consequence, the best relay selection criterion is found as

$$
\begin{aligned}
\text { Best relay } & =\arg \max _{i \in D_{m}} \operatorname{SINR}_{\mathrm{d}}\left(D=D_{m}, \mathrm{CR}_{i}\right) \\
& =\arg \max _{i \in D_{m}}\left|h_{i d}(k)\right|^{2}
\end{aligned}
$$

It is worth mentioning that using the best relay selection criterion as given above, we can further develop a specific relay selection algorithm in a centralized or a distributed fashion. To be specific, for a centralized relay selection strategy, the cognitive source should maintain a table that consists of all the cognitive relays and the related channel information (i.e., $\left|h_{i d}(k)\right|^{2}$ ). Note that such channel information could be estimated by the cognitive relays and then forwarded to the cognitive source over a feedback channel. After that, the best cognitive relay can be easily determined by looking up the table, which is called a centralized relay selection approach. For a distributed relay selection strategy, each cognitive relay should maintain a timer [19] and set an initial value of the timer in inverse proportional to $\left|h_{i d}(k)\right|^{2}$ as given by Eq. (16), resulting in the best cognitive relay with the smallest initial value for its timer. Hence, the best cognitive relay exhausts its timer earliest compared with the other relays, and then broadcasts a control packet to notify the cognitive source and other relays [19]. Hence, in the given case $D=D_{m}$, the received SINR at CD is given by

$$
\operatorname{SINR}_{\mathrm{d}}\left(D=D_{m}\right)=\max _{i \in D_{m}} \operatorname{SINR}_{\mathrm{d}}\left(D=D_{m}, \mathrm{CR}_{i}\right)
$$


where $\operatorname{SINR}_{\mathrm{d}}\left(D=D_{m}, \mathrm{CR}_{i}\right)$ is given by Eq. (15). This completes the model formulation for the SFSS-BRDT scheme. We next explain the signal modeling for the FFSS-BRDT scheme in the following subsection.

2) Modeling of the FFSS-BRDT scheme: As can be seen from the previous subsection, the difference between the SFSS-BRDT and the FFSS-BRDT schemes lies the spectrum sensing part. Thus, we only need to model the sensing phase of the FFSS-BRDT scheme, since its transmission phase is identical to the SFSS-BRDT scheme as modeled by Eqs. (6) - (16). For the FFSS strategy, only a forward error correction code (without an additional error detection code) is utilized by CRs to encode their initial sensing results, leading to an encoded bit stream that is modulated to a constellation symbol denoted by $z_{i}(k, 2)$. After that, CRs forward the modulated symbols to CS over $M$ orthogonal common control subchannels. Thus, the received signals at CS from CRs can be represented as

$$
y_{s}^{i}(k, 2)=\sqrt{P_{s}} h_{i s}(k) z_{i}(k, 2)+n_{s}^{i}(k, 2), \quad i \in \mathcal{R}
$$

Then, CS will attempt to decode $\hat{H}_{i}(k, 1)$ based on its received signal and the decoded outcome is denoted by $\hat{H}_{s}^{i}(k, 2)$, which can be given by

$$
\hat{H}_{s}^{i}(k, 2)= \begin{cases}\hat{H}_{i}(k, 1), & \Theta_{i s}(k, 2)=0 \\ \operatorname{rand}\left\{H_{0}, H_{1}\right\}, & \Theta_{i s}(k, 2)=1\end{cases}
$$

where $\operatorname{rand}\left\{H_{0}, H_{1}\right\}$ indicates the equiprobable selection of an element from $\left\{H_{0}, H_{1}\right\}, \Theta_{i s}(k, 2)=0$ denotes that no outage occurs over the channel from $\mathrm{CR}_{i}$ to $\mathrm{CS}$, and $\Theta_{i s}(k, 2)=1$ denotes an outage event occurring over the corresponding channel. Finally, CS combines $\hat{H}_{s}(k, 1)$ and $\hat{H}_{s}^{i}(k, 2)$ through a logical AND rule. Hence, the final spectrum sensing result at CS, $\hat{H}_{s}(k)$, is expressed as

$$
\hat{H}_{s}(k)=\hat{H}_{s}(k, 1) \underset{i=1}{\stackrel{Q}{\otimes}} \hat{H}_{s}^{i}(k, 2)
$$

We now have completed the signal modeling for both the SFSS-BRDT and the FFSS-BRDT schemes. In what follows, we focus on their performance analysis in Rayleigh fading environments.

\section{Performance Analysis of the SFSS-BRDT and FFSS-BRDT SCHEMES OVER RAYLEIGH FADING CHANNELS}

In this section, we first derive a closed-form outage probability expression with a primary user protection constraint for the SFSS-BRDT scheme over Rayleigh fading channels. Based on the derived outage probability, a performance metric, referred to as spectrum hole utilization efficiency, is defined and used to quantify the percentage of spectrum holes utilized by the cognitive source for its successful data transmissions without channel outage. Finally, we also present the spectrum hole utilization efficiency analysis for the FFSS-BRDT scheme to make a performance comparison with the SFSS-BRDT scheme.

\section{A. Analysis of The SFSS-BRDT Scheme}

Following [14], [16], an outage event is considered to occur when channel capacity falls below data transmission rate $R$. Thus, the outage probability of the SFSS-BRDT scheme can be calculated as

$$
\begin{aligned}
\mathrm{P}_{\text {out }}= & \operatorname{Pr}\left\{\frac{1-\alpha}{2} \log _{2}\left(1+\operatorname{SINR}_{\mathrm{d}}\right)<R\right\} \\
= & \operatorname{Pr}\left\{\operatorname{SINR}_{\mathrm{d}}(D=\emptyset)<\gamma_{s} \Delta, D=\emptyset\right\} \\
& +\sum_{m=1}^{2^{M}-1} \operatorname{Pr}\left\{\operatorname{SINR}_{\mathrm{d}}\left(D=D_{m}\right)<\gamma_{s} \Delta, D=D_{m}\right\}
\end{aligned}
$$

where $\Delta=\left[2^{2 R /(1-\alpha)}-1\right] / \gamma_{s}, \operatorname{SINR}_{\mathrm{d}}(D=\emptyset)$ and $\operatorname{SINR}_{\mathrm{d}}\left(D=D_{m}\right)$ are given by Eq. (12) and Eq. (17), respectively. Note that the factor $1 / 2$ in the first equation of Eq. (19) is resulted from a half-duplex relay constraint [14], [16]. According to Eq. (10) and Eq. (12), the term $\operatorname{Pr}\left\{\operatorname{SINR}_{\mathrm{d}}(D=\emptyset)<\gamma_{s} \Delta, \quad D=\emptyset\right\}$ in Eq. (21) can be expanded as

$$
\begin{aligned}
\operatorname{Pr} & \left\{\operatorname{SINR}_{\mathrm{d}}(D=\emptyset)<\gamma_{s} \Delta, D=\emptyset\right\} \\
= & \mathrm{P}_{\mathrm{a}} \operatorname{Pd}_{s} \operatorname{Pr}\left\{\left|h_{s d}(k)\right|^{2}<\Delta\right\} \prod_{i=1}^{M} \operatorname{Pr}\left\{\left|h_{s i}(k)\right|^{2}<\Delta\right\} \\
& +\left(1-\mathrm{P}_{\mathrm{a}}\right) \operatorname{Pf}_{s} \operatorname{Pr}\left\{\left|h_{s d}(k)\right|^{2}-\left|h_{p d}(k)\right|^{2} \gamma_{p} \Delta<\Delta\right\} \\
& \times \prod_{i=1}^{M} \operatorname{Pr}\left\{\left|h_{s i}(k)\right|^{2}-\left|h_{p i}(k)\right|^{2} \gamma_{p} \Delta<\Delta\right\} \\
& +\mathrm{P}_{\mathrm{a}}\left(1-\operatorname{Pd}_{s}\right)+\left(1-\mathrm{P}_{\mathrm{a}}\right)\left(1-\operatorname{Pf}_{s}\right)
\end{aligned}
$$

where $\mathrm{P}_{\mathrm{a}}=\operatorname{Pr}\left\{H_{p}(k)=H_{0}\right\}$ is the probability that there is a spectrum hole, $\operatorname{Pd}_{s}=\operatorname{Pr}\left\{\hat{H}_{s}(k)=H_{0} \mid H_{p}(k)=H_{0}\right\}$ and $\operatorname{Pf}_{s}=\operatorname{Pr}\left\{\hat{H}_{s}(k)=H_{0} \mid H_{p}(k)=H_{1}\right\}$ are, respectively, the probability of detection and false alarm of spectrum holes at CS. Notice that random variables (RVs) $\left|h_{s d}(k)\right|^{2},\left|h_{s i}(k)\right|^{2}$, $\left|h_{p d}(k)\right|^{2}$ and $\left|h_{p i}(k)\right|^{2}$ follow exponential distributions with means $\sigma_{s d}^{2}, \sigma_{s i}^{2}, \sigma_{p d}^{2}$ and $\sigma_{p i}^{2}$, respectively. Moreover, the difference between two identically and exponentially distributed random variables has a Laplace distribution. Therefore, the probabilities as given in Eq. (22) are calculated as

$$
\begin{aligned}
& \operatorname{Pr}\left\{\left|h_{s d}(k)\right|^{2}<\Delta\right\}=1-\exp \left(-\frac{\Delta}{\sigma_{s d}^{2}}\right) \\
& \operatorname{Pr}\left\{\left|h_{s i}(k)\right|^{2}<\Delta\right\}=1-\exp \left(-\frac{\Delta}{\sigma_{s i}^{2}}\right) \\
& \operatorname{Pr}\left\{\left|h_{s d}(k)\right|^{2}-\left|h_{p d}(k)\right|^{2} \gamma_{p} \Delta<\Delta\right\} \\
& =1-\frac{\sigma_{s d}^{2}}{\sigma_{p d}^{2} \gamma_{p} \Delta+\sigma_{s d}^{2}} \exp \left(-\frac{\Delta}{\sigma_{s d}^{2}}\right) \\
& \operatorname{Pr}\left\{\left|h_{s i}(k)\right|^{2}-\left|h_{p i}(k)\right|^{2} \gamma_{p} \Delta<\Delta\right\} \\
& =1-\frac{\sigma_{s i}^{2}}{\sigma_{p i}^{2} \gamma_{p} \Delta+\sigma_{s i}^{2}} \exp \left(-\frac{\Delta}{\sigma_{s i}^{2}}\right)
\end{aligned}
$$


From Eq. (13) and Eq. (17), the term $\operatorname{Pr}\left\{\operatorname{SINR}_{\mathrm{d}}\left(D=D_{m}\right)<\right.$ $\left.\gamma_{s} \Delta, D=D_{m}\right\}$ in Eq. (21) is found as

$$
\begin{aligned}
\operatorname{Pr} & \left\{\operatorname{SINR}_{\mathrm{d}}\left(D=D_{m}\right)<\gamma_{s} \Delta, D=D_{m}\right\} \\
= & \mathrm{P}_{\mathrm{a}} \operatorname{Pd}_{s} \operatorname{Pr}\left\{\max _{i \in D_{m}}\left|h_{i d}(k)\right|^{2}<2 \Delta-\left|h_{s d}(k)\right|^{2}\right\} \\
& \times \prod_{i \in D_{m}} \operatorname{Pr}\left\{\left|h_{s i}(k)\right|^{2}>\Delta\right\} \prod_{j \in \bar{D}_{m}} \operatorname{Pr}\left\{\left|h_{s j}(k)\right|^{2}<\Delta\right\} \\
+ & \left(1-\mathrm{P}_{\mathrm{a}}\right) \operatorname{Pf}_{s} \prod_{i \in D_{m}} \operatorname{Pr}\left\{\left|h_{s i}(k)\right|^{2}-\left|h_{p i}(k)\right|^{2} \gamma_{p} \Delta>\Delta\right\} \\
& \times \prod_{j \in \bar{D}_{m}} \operatorname{Pr}\left\{\left|h_{s j}(k)\right|^{2}-\left|h_{p j}(k)\right|^{2} \gamma_{p} \Delta<\Delta\right\} \\
& \times \operatorname{Pr}\left\{\max _{i \in D_{m}}\left|h_{i d}(k)\right|^{2}<2 \Delta-\left|h_{s d}(k)\right|^{2}+2\left|h_{p d}(k)\right|^{2} \gamma_{p} \Delta\right\}
\end{aligned}
$$

wherein

$$
\begin{aligned}
& \operatorname{Pr}\left\{\left|h_{s i}(k)\right|^{2}>\Delta\right\}=\exp \left(-\frac{\Delta}{\sigma_{s i}^{2}}\right) \\
& \operatorname{Pr}\left\{\left|h_{s j}(k)\right|^{2}<\Delta\right\}=1-\exp \left(-\frac{\Delta}{\sigma_{s j}^{2}}\right) \\
& \operatorname{Pr}\left\{\left|h_{s i}(k)\right|^{2}-\left|h_{p i}(k)\right|^{2} \gamma_{p} \Delta>\Delta\right\} \\
& =\frac{\sigma_{s i}^{2}}{\sigma_{p i}^{2} \gamma_{p} \Delta+\sigma_{s i}^{2}} \exp \left(-\frac{\Delta}{\sigma_{s i}^{2}}\right) \\
& \operatorname{Pr}\left\{\left|h_{s j}(k)\right|^{2}-\left|h_{p j}(k)\right|^{2} \gamma_{p} \Delta<\Delta\right\} \\
& =1-\frac{\sigma_{s j}^{2}}{\sigma_{p j}^{2} \gamma_{p} \Delta+\sigma_{s j}^{2}} \exp \left(-\frac{\Delta}{\sigma_{s j}^{2}}\right)
\end{aligned}
$$

Moreover, the probability $\operatorname{Pr}\left\{\max _{i \in D_{m}}\left|h_{i d}(k)\right|^{2}<2 \Delta-\right.$ $\left.\left|h_{s d}(k)\right|^{2}\right\}$ in Eq. (24) is calculated as

$$
\begin{aligned}
& \operatorname{Pr}\left\{\max _{i \in D_{m}}\left|h_{i d}(k)\right|^{2}<2 \Delta-\left|h_{s d}(k)\right|^{2}\right\} \\
& =\int_{0}^{2 \Delta} \frac{1}{\sigma_{s d}^{2}} \exp \left(-\frac{x}{\sigma_{s d}^{2}}\right) \prod_{i \in D_{m}}\left[1-\exp \left(-\frac{2 \Delta-x}{\sigma_{i d}^{2}}\right)\right] d x
\end{aligned}
$$

By using the binomial expansion formula, the term $\prod_{i \in D_{m}}\left[1-\exp \left(-\frac{2 \Delta-x}{\sigma_{i d}^{2}}\right)\right]$ can be expressed as $1+\sum_{n=1}^{2^{\left|D_{m}\right|}-1}(-1)^{\left|S_{m}(n)\right|} \exp \left(-\sum_{i \in S_{m}(n)} \frac{2 \Delta-x}{\sigma_{i d}^{2}}\right), \quad$ where

$\left|D_{m}\right|$ is the number of the elements in decoding set $D_{m}$ and $S_{m}(n)$ is the $n$-th non-empty subcollection of the elements in $D_{m}$. Substituting this result into the preceding equation and performing the integration yield

$$
\begin{aligned}
& \operatorname{Pr}\left\{\max _{i \in D_{m}}\left|h_{i d}(k)\right|^{2}<2 \Delta-\left|h_{s d}(k)\right|^{2}\right\}=1-\exp \left(-\frac{2 \Delta}{\sigma_{s d}^{2}}\right) \\
& \quad+\sum_{n=1}^{2^{\left|D_{m}\right|}-1}(-1)^{\left|S_{m}(n)\right|} \phi\left[\sigma_{s d}^{2}, \sigma_{i d}^{2}, S_{m}(n)\right]
\end{aligned}
$$

where $\phi\left[\sigma_{s d}^{2}, \sigma_{i d}^{2}, S_{m}(n)\right]$ is given by

$$
\phi\left[\sigma_{s d}^{2}, \sigma_{i d}^{2}, S_{m}(n)\right]=\left\{\begin{array}{l}
\frac{2 \Delta}{\sigma_{s d}^{2}} \exp \left(-\frac{2 \Delta}{\sigma_{s d}^{2}}\right), \quad \sum_{i \in S_{m}(n)} \frac{1}{\sigma_{i d}^{2}}=\frac{1}{\sigma_{s d}^{2}} \\
\frac{\exp \left(-\sum_{i \in S_{m}(n)} \frac{2 \Delta}{\sigma_{i d}^{2}}\right)-\exp \left(-\frac{2 \Delta}{\sigma_{s d}^{2}}\right)}{1-\sum_{i \in S_{m}(n)} \frac{\sigma_{s d}^{2}}{\sigma_{i d}^{2}}}, \text { others }
\end{array}\right.
$$

Besides, the probability $\operatorname{Pr}\left\{\max _{i \in D_{m}}\left|h_{i d}(k)\right|^{2}<2 \Delta-\right.$ $\left.\left|h_{s d}(k)\right|^{2}+2\left|h_{p d}(k)\right|^{2} \gamma_{p} \Delta\right\}$ in Eq. (24) is given by (see Appendix A for details)

$$
\begin{aligned}
& \operatorname{Pr}\left\{\max _{i \in D_{m}}\left|h_{i d}(k)\right|^{2}<2 \Delta-\left|h_{s d}(k)\right|^{2}+2\left|h_{p d}(k)\right|^{2} \gamma_{p} \Delta\right\} \\
& =\mathrm{A}_{m}+\mathrm{B}_{m}
\end{aligned}
$$

where the closed-form expressions of $\mathrm{A}_{m}$ and $\mathrm{B}_{m}$ are given by Eq. (A.6) and Eq. (A.7), respectively, in Appendix A. Now, we start to analyze $\mathrm{Pd}_{s}$ and $\mathrm{Pf}_{s}$ as given in Eq. (22) and Eq. (24). Considering Eqs. (5) and (7), an overall probability of detection of spectrum holes at $\mathrm{CS}\left(\mathrm{Pd}_{s}\right)$, called detection probability, can be calculated as Eq. (29), where $\operatorname{Pd}_{s, 1}=\operatorname{Pr}\left\{\hat{H}_{s}(k, 1)=H_{0} \mid H_{p}(k)=H_{0}\right\}$ and $\operatorname{Pd}_{i, 1}=$ $\operatorname{Pr}\left\{\hat{H}_{i}(k, 1)=H_{0} \mid H_{p}(k)=H_{0}\right\}$ are the individual probabilities of detection of spectrum holes at $\mathrm{CS}$ and $\mathrm{CR}_{i}$, respectively. Similarly, an overall probability of false alarm of spectrum holes at CS $\left(\mathrm{Pf}_{s}\right)$, called false alarm probability, is given by Eq. (30), where $\operatorname{Pf}_{s, 1}=\operatorname{Pr}\left\{\hat{H}_{s}(k, 1)=H_{0} \mid H_{p}(k)=H_{1}\right\}$ and $\operatorname{Pf}_{i, 1}=\operatorname{Pr}\left\{\hat{H}_{i}(k, 1)=H_{0} \mid H_{p}(k)=H_{1}\right\}$ are the individual probabilities of false alarm of spectrum holes at $\mathrm{CS}$ and $\mathrm{CR}_{i}$, respectively. Note that, throughout this paper, an energy detector [6], [10] is considered in evaluating the performance of the spectrum sensing phase. Following [10], $\mathrm{Pd}_{s, 1}$ and $\mathrm{Pf}_{s, 1}$ can be represented from Eq. (1) as

$$
\begin{aligned}
\operatorname{Pd}_{s, 1} & =\operatorname{Pr}\left\{\hat{H}_{s}(k, 1)=H_{0} \mid H_{p}(k)=H_{0}\right\} \\
& =\operatorname{Pr}\left\{\left|n_{s}(k, 1)\right|^{2}<\delta\right\}
\end{aligned}
$$

and

$$
\begin{aligned}
\operatorname{Pf}_{s, 1} & =\operatorname{Pr}\left\{\hat{H}_{s}(k, 1)=H_{0} \mid H_{p}(k)=H_{1}\right\} \\
& =\operatorname{Pr}\left\{\frac{\alpha}{2} P_{p}\left|h_{p s}(k)\right|^{2}+\left|n_{s}(k, 1)\right|^{2}<\delta\right\}
\end{aligned}
$$

where $\delta$ is an energy detection threshold. From Eq. (31) and Eq. (32), we can obtain

$$
\operatorname{Pf}_{s, 1}= \begin{cases}1-\left[1-\ln \left(1-\mathrm{Pd}_{s, 1}\right)\right]\left(1-\mathrm{Pd}_{s, 1}\right), & \alpha \sigma_{p s}^{2} \gamma_{p}=4 \\ 1-\frac{4\left(1-\mathrm{Pd}_{s, 1}\right)}{4-\alpha \sigma_{p s}^{2} \gamma_{p}} & \\ -\frac{\alpha \sigma_{p s}^{2} \gamma_{p}}{\alpha \sigma_{p s}^{2} \gamma_{p}-4}\left(1-\mathrm{Pd}_{s, 1}\right)^{\frac{4}{\alpha \sigma_{p s}^{2} \gamma_{p}}}, & \text { otherwise }\end{cases}
$$

Similar to the derivation of Eq. (33) and following Eq. (2), we obtain

$$
\operatorname{Pf}_{i, 1}= \begin{cases}1-\left[1-\ln \left(1-\mathrm{Pd}_{i, 1}\right)\right]\left(1-\mathrm{Pd}_{i, 1}\right), & \alpha \sigma_{p i}^{2} \gamma_{p}=4 \\ 1-\frac{4\left(1-\mathrm{Pd}_{i, 1}\right)}{4-\alpha \sigma_{p i}^{2} \gamma_{p}} & \\ -\frac{\alpha \sigma_{p i}^{2} \gamma_{p}}{\alpha \sigma_{p i}^{2} \gamma_{p}-4}\left(1-\mathrm{Pd}_{i, 1}\right)^{\frac{4}{\alpha \sigma_{p i}^{2} \gamma_{p}}}, & \text { otherwise }\end{cases}
$$

In addition, $\operatorname{Pr}(C=\emptyset)$ and $\operatorname{Pr}\left(C=C_{m}\right)$ are determined by

$$
\operatorname{Pr}(C=\emptyset)=\prod_{i=1}^{M}\left[1-\exp \left(-\frac{\Lambda}{\sigma_{i s}^{2}}\right)\right]
$$




$$
\begin{aligned}
\operatorname{Pd}_{s}= & \operatorname{Pr}\left\{\hat{H}_{s}(k)=H_{0} \mid H_{p}(k)=H_{0}\right\} \\
= & \operatorname{Pr}\left\{\hat{H}_{s}(k)=H_{0} \mid H_{p}(k)=H_{0}, C=\emptyset\right\} \operatorname{Pr}\left(C=\emptyset \mid H_{p}(k)=H_{0}\right) \\
& +\sum_{m=1}^{2^{M}-1} \operatorname{Pr}\left\{\hat{H}_{s}(k)=H_{0} \mid H_{p}(k)=H_{0}, C=C_{m}\right\} \operatorname{Pr}\left(C=C_{m} \mid H_{p}(k)=H_{0}\right) \\
= & \operatorname{Pr}(C=\emptyset) \operatorname{Pd}_{s, 1}+\sum_{m=1}^{2^{M}-1} \operatorname{Pr}\left(C=C_{m}\right) \operatorname{Pd} d_{s, 1} \prod_{i \in C_{m}} \operatorname{Pd}_{i, 1} \\
\operatorname{Pf}_{s}= & \operatorname{Pr}\left\{\hat{H}_{s}(k)=H_{0} \mid H_{p}(k)=H_{1}\right\} \\
= & \operatorname{Pr}\left\{\hat{H}_{s}(k)=H_{0} \mid H_{p}(k)=H_{1}, C=\emptyset\right\} \operatorname{Pr}\left(C=\emptyset \mid H_{p}(k)=H_{1}\right) \\
& +\sum_{m=1}^{2^{M}-1} \operatorname{Pr}\left\{\hat{H}_{s}(k)=H_{0} \mid H_{p}(k)=H_{1}, C=C_{m}\right\} \operatorname{Pr}\left(C=C_{m} \mid H_{p}(k)=H_{1}\right) \\
& \operatorname{Pr}(C=\emptyset) \operatorname{Pf}_{s, 1}+\sum_{m=1}^{2^{M}-1} \operatorname{Pr}\left(C=C_{m}\right) \operatorname{Pf}_{s, 1} \prod_{i \in C_{m}} \operatorname{Pf}_{i, 1}
\end{aligned}
$$

and

$$
\operatorname{Pr}\left(C=C_{m}\right)=\prod_{i \in C_{m}} \exp \left(-\frac{\Lambda}{\sigma_{i s}^{2}}\right) \prod_{j \in \bar{C}_{m}}\left[1-\exp \left(-\frac{\Lambda}{\sigma_{j s}^{2}}\right)\right]
$$

where $\Lambda=\left[2^{2 M /\left(\alpha B_{c} T_{c}\right)}-1\right] / \gamma_{s}$. Clearly, if there is a false alarm of spectrum holes, the CS will start its data traffic transmissions and thus interferes with the primary user. Accordingly, for the protection of the primary user, the overall false alarm probability shall be guaranteed to be below a required target value. Given a target value $\mathrm{Pf}_{s, \text { thr }}$ and assuming that the sensing performance at $\mathrm{CS}$ associated with each possible set $C$ is guaranteed with the same false alarm requirement, we can obtain solutions from Eq. (30) as $\mathrm{Pf}_{s, 1}=\left(\mathrm{Pf}_{s, \text { thr }}\right)^{1 /(|C|+1)}$ and $\mathrm{Pf}_{i, 1}=\left(\mathrm{Pf}_{s, \text { thr }}\right)^{1 /(|C|+1)}$, respectively, where $|C|$ is the number of CRs in set $C$. Substituting $\operatorname{Pf}_{s, 1}=\left(\mathrm{Pf}_{s, \text { thr }}\right)^{1 /(|C|+1)}$ and $\mathrm{Pf}_{i, 1}=\left(\mathrm{Pf}_{s, \text { thr }}\right)^{1 /(|C|+1)}$ into Eqs. (33) and (34), we can obtain the numerical solutions to $\mathrm{Pd}_{s, 1}$ and $\mathrm{Pd}_{i, 1}$, by using which an overall detection probability $\mathrm{Pd}_{s}$ is determined from Eq. (29) under a given target false alarm probability $\mathrm{Pf}_{s, \text { thr }}$.

Now, we have completed the analysis of spectrum sensing performance for the SFSS-BRDT scheme. Now, we have derived a closed-form expression of the outage probability for the SFSS-BRDT scheme. Based on the derived outage probability, a spectrum hole utilization efficiency is defined as

$$
\eta=\frac{1-\mathrm{P}_{\text {out }}}{\mathrm{P}_{\mathrm{a}}}
$$

where $1-\mathrm{P}_{\text {out }}$ indicates the quantity of spectrum holes that are utilized by the cognitive source (CS) for its successful data transmissions (without channel outage) and $\mathrm{P}_{\mathrm{a}}$ implies the total quantity of spectrum holes available for CS. Accordingly, $\left(1-\mathrm{P}_{\text {out }}\right) / \mathrm{P}_{\mathrm{a}}$ can be seen as a measure to quantify the percentage of spectrum holes utilized by CS for its successful data transmission. Furthermore, combining Eq. (21) and Eq. (37), one can see that $\alpha$ is a parameter that can be optimized to maximize the spectrum hole utilization efficiency. Obtaining a general expression for optimal spectrum sensing overhead, $\alpha_{\mathrm{opt}}$, as a function of other parameters is challenging. Nev- ertheless, the optimal value $\alpha_{\text {opt }}$ can be determined through numerical calculations.

\section{B. Analysis of The FFSS-BRDT Scheme}

For the purpose of performance comparison, we also examine the spectrum hole utilization efficiency of the FFSS-BRDT scheme. Since the difference between the SFSS-BRDT and FFSS-BRDT schemes lies the spectrum sensing phase only, we just need to investigate the spectrum sensing performance of the FFSS-BRDT scheme. From Eq. (20), the overall probability of detection of spectrum holes at CS using FFSS-BRDT scheme can be calculated as

$$
\begin{aligned}
\operatorname{Pd}_{s} & =\operatorname{Pr}\left\{\hat{H}_{s}(k)=H_{0} \mid H_{p}(k)=H_{0}\right\} \\
& =\operatorname{Pr}\left\{\hat{H}_{s}(k, 1){\left.\underset{i=1}{M} \hat{H}_{s}^{i}(k, 2)=H_{0} \mid H_{p}(k)=H_{0}\right\}}=\operatorname{Pd}_{s, 1} \prod_{i=1}^{M} \operatorname{Pd}_{s, 2}^{i}\right.
\end{aligned}
$$

where $\operatorname{Pd}_{s, 2}^{i}=\operatorname{Pr}\left\{\hat{H}_{s}^{i}(k, 2)=H_{0} \mid H_{p}(k)=H_{0}\right\}$. Similarly, from Eq. (20), the probability of false alarm of spectrum holes at CS is given by

$$
\begin{aligned}
& \operatorname{Pf}_{s}=\operatorname{Pr}\left\{\hat{H}_{s}(k)=H_{0} \mid H_{p}(k)=H_{1}\right\} \\
& =\operatorname{Pr}\left\{\hat{H}_{s}(k, 1) \underset{i=1}{\stackrel{M}{\otimes}} \hat{H}_{s}^{i}(k, 2)=H_{0} \mid H_{p}(k)=H_{1}\right\} \\
& =\operatorname{Pf}_{s, 1} \prod_{i=1}^{M} \operatorname{Pf}_{s, 2}^{i}
\end{aligned}
$$

where $\operatorname{Pf}_{s, 2}^{i}=\operatorname{Pr}\left\{\hat{H}_{s}^{i}(k, 2)=H_{0} \mid H_{p}(k)=H_{1}\right\}$. Notice that the relationship between $\operatorname{Pd}_{s, 1}$ and $\mathrm{Pf}_{s, 1}$ is described by Eq. (33). Moreover, substituting $\hat{H}_{s}^{i}(k, 2)$ from Eq. (19) into $\mathrm{Pd}_{s, 2}^{i}$ and $\mathrm{Pf}_{s, 2}^{i}$ yields

$$
\begin{aligned}
& \operatorname{Pd}_{s, 2}^{i}=\operatorname{Pr}\left\{\hat{H}_{i}(k, 1)=H_{0} \mid H_{p}(k)=H_{0}\right\} \operatorname{Pr}\left\{\Theta_{i s}(k, 2)=0\right\} \\
& +\operatorname{Pr}\left\{\operatorname{rand}\left\{H_{0}, H_{1}\right\}=H_{0} \mid H_{p}(k)=H_{0}\right\} \operatorname{Pr}\left\{\Theta_{i s}(k, 2)=1\right\} \\
& =\operatorname{Pd}_{i, 1} \operatorname{Pr}\left\{\Theta_{i s}(k, 2)=0\right\}+\frac{1}{2} \operatorname{Pr}\left\{\Theta_{i s}(k, 2)=1\right\}
\end{aligned}
$$


and

$$
\begin{aligned}
& \operatorname{Pf}_{s, 2}^{i}=\operatorname{Pr}\left\{\hat{H}_{i}(k, 1)=H_{0} \mid H_{p}(k)=H_{1}\right\} \operatorname{Pr}\left\{\Theta_{i s}(k, 2)=0\right\} \\
& +\operatorname{Pr}\left\{\operatorname{rand}\left\{H_{0}, H_{1}\right\}=H_{0} \mid H_{p}(k)=H_{1}\right\} \operatorname{Pr}\left\{\Theta_{i s}(k, 2)=1\right\} \\
& =\operatorname{Pf}_{i, 1} \operatorname{Pr}\left\{\Theta_{i s}(k, 2)=0\right\}+\frac{1}{2} \operatorname{Pr}\left\{\Theta_{i s}(k, 2)=1\right\}
\end{aligned}
$$

where the false alarm probability, $\mathrm{Pf}_{i, 1}$, is depicted as a function of the detection probability, $\mathrm{Pd}_{i, 1}$, as given by Eq. (34). It is noted that an initial spectrum sensing result $\hat{H}_{i}(k, 1)$ carries 1-bit information only in an information-theoretic sense and the time-bandwidth product of the common control channel is $B_{c} T_{c}$. Thus, the transmission rate of such an initial sensing result over the common control channel is given by $1 /\left(B_{c} T_{c}\right)$. Moreover, as mentioned in the paragraph below Eq. (19), $\Theta_{i s}(k, 2)=0$ denotes that no outage event occurs over the channel from the $\mathrm{CR}_{i}$ to CS. In addition, from Eq. (18), the probability of occurrence of $\Theta_{i s}(k, 2)=0$ is calculated as

$$
\begin{aligned}
\operatorname{Pr}\left\{\Theta_{i s}(k, 2)=0\right\} & =\operatorname{Pr}\left\{\frac{\alpha}{2 M} \log _{2}\left(1+\left|h_{i s}(k)\right|^{2} \gamma_{s}\right)>\frac{1}{B_{c} T_{c}}\right\} \\
& =\exp \left(-\frac{\Lambda}{\sigma_{i s}^{2}}\right)
\end{aligned}
$$

where $\Lambda=\left[2^{2 M /\left(\alpha B_{c} T_{c}\right)}-1\right] / \gamma_{s}$. From Eq. (42), $\operatorname{Pr}\left\{\Theta_{i s}(k, 2)=1\right\}$ is easily found as $1-\operatorname{Pr}\left\{\Theta_{i s}(k, 2)=\right.$ $0\}$. Hence, given a target value $\mathrm{Pf}_{s, \text { thr }}$ and assuming each cognitive user with the same detection performance, we can obtain solutions from Eq. (39) as $\mathrm{Pf}_{s, 1}=\left(\mathrm{Pf}_{s, \text { thr }}\right)^{1 /(M+1)}$ and $\mathrm{Pf}_{s, 2}^{i}=\left(\mathrm{Pf}_{s, \text { thr }}\right)^{1 /(M+1)}$, respectively. Substituting $\mathrm{Pf}_{s, 1}=\left(\mathrm{Pf}_{s, \mathrm{thr}}\right)^{1 /(M+1)}$ into Eq. (33) easily obtains the corresponding numerical solution to $\mathrm{Pd}_{s, 1}$. Meanwhile, applying $\mathrm{Pf}_{s, 2}^{i}=\left(\mathrm{Pf}_{s, \text { thr }}\right)^{1 /(M+1)}$ to Eq. (41) yields $\mathrm{Pf}_{i, 1}=$ $\exp \left(\frac{\Lambda}{\sigma_{i s}^{2}}\right)\left(\mathrm{Pf}_{s, \text { thr }}\right)^{1 /(M+1)}-\frac{1}{2}\left[\exp \left(\frac{\Lambda}{\sigma_{i s}^{2}}\right)-1\right]$ and the corresponding detection probability $\mathrm{Pd}_{i, 1}$ can be found from Eq. (34), by using which a numerical $\mathrm{Pd}_{s, 2}^{i}$ is determined from Eq. (40). Finally, substituting the results $\mathrm{Pd}_{s, 1}$ and $\mathrm{Pd}_{s, 2}^{i}$ into Eq. (38), we can obtain an overall detection probability $\mathrm{Pd}_{s}$ under a given target false alarm probability $\mathrm{Pf}_{s, \text { thr }}$. Now, we have completed the analysis of spectrum sensing performance for the FFSS-BRDT scheme. Note that the outage probability analysis of the FFSS-BRDT scheme differs from that of the SFSS-BRDT scheme only in the terms $\mathrm{Pd}_{s}$ and $\mathrm{Pf}_{s}$. Therefore, a closed-form expression of the spectrum hole utilization efficiency for the FFSS-BRDT scheme can be obtained from Eq. (37) with the replacement of Eqs. (29) and (30) by Eqs. (38) and (39), respectively.

\section{Numerical and Simulation Results}

In this section, we conduct numerical evaluations for the SFSS-BRDT and the FFSS-BRDT schemes, showing the advantages of the former scheme. Notice that the primary user will be interfered by the cognitive users when a false alarm of spectrum holes occurs at CS. Therefore, the false alarm probability $\mathrm{Pf}_{s}$ shall be set to be below a required value by the cognitive system to guarantee a primary qualityof-service (QoS) requirement. We use $\mathrm{Pf}_{s}=10^{-3}$ in the following numerical evaluations. In addition, we demonstrate

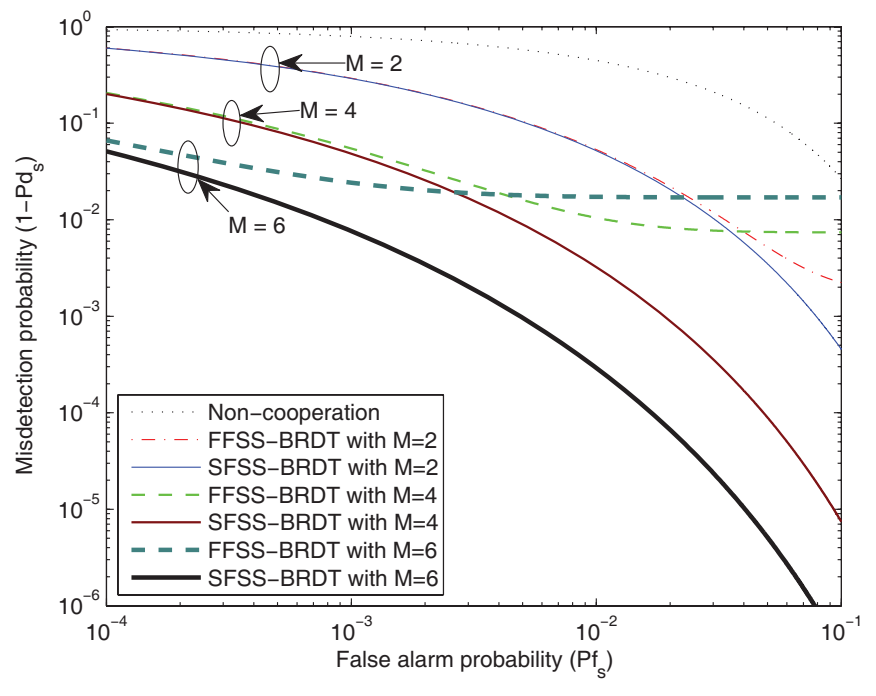

Fig. 3. The misdetection probability $\left(1-\mathrm{Pd}_{s}\right)$ versus the false alarm probability $\left(\mathrm{Pf}_{s}\right)$ of the non-cooperation, the SFSS-BRDT and the FFSS-BRDT schemes for the different number of cognitive relays $M$ with $\mathrm{P}_{\mathrm{a}}=0.6$, $\gamma_{p}=20 \mathrm{~dB}, \alpha=0.5, B_{c} T_{c}=100$, and $\sigma_{p s}^{2}=\sigma_{p i}^{2}=\sigma_{i s}^{2}=1$.

that a maximum spectrum hole utilization efficiency can be achieved through an optimization of the spectrum sensing overhead.

Fig. 3 depicts the misdetection probability $\left(1-\mathrm{Pd}_{s}\right)$ versus the false alarm probability $\left(\mathrm{Pf}_{s}\right)$ of the SFSS-BRDT and the FFSS-BRDT schemes. The performance curve of the non-cooperation scheme is also plotted in this figure. From Fig. 3, one can see that the SFSS-BRDT scheme performs better than both the FFSS-BRDT and the non-cooperation schemes. It is also shown from Fig. 3 that the misdetection probability curves of the FFSS-BRDT scheme corresponding to $M=2,4$ and 6 converge to their floors, respectively, in high false alarm probability regions. As the number of cognitive relays increases from $M=2$ to 6 , the misdetection probability floor increases. This is because that the channel outages will occur during the transmission of initial spectrum sensing results from CRs to $\mathrm{CS}$, which results in the initial sensing results received at $\mathrm{CS}$ in error. Since the FFSSBRDT scheme can not recognize such errors, it in turn impairs the fusion performance of the FFSS-BRDT scheme. Moreover, the occurrence of these errors is independent of the false alarm probability and becomes the dominant factor to adversely affect the sensing performance in high false alarm probability regions, thus causing a misdetection probability floor for the FFSS-BRDT scheme. In contrast, the SFSSBRDT scheme is able to identify and discard these errors by CRC checking, thus no misdetection probability floor occurs for the SFSS-BRDT scheme. In addition, with an increased number of cognitive relays and under fixed/limited common control channel resources, the probability of occurrence of such an error increases due to the fewer common control channel resources assigned for each CR, which finally leads to an increase of the misdetection probability floor for the FFSS-BRDT scheme.

In Fig. 4, we show the detection probability versus the number of CRs of the SFSS-BRDT and the FFSS-BRDT schemes with a required false alarm probability $\mathrm{Pf}_{s}=10^{-3}$. 


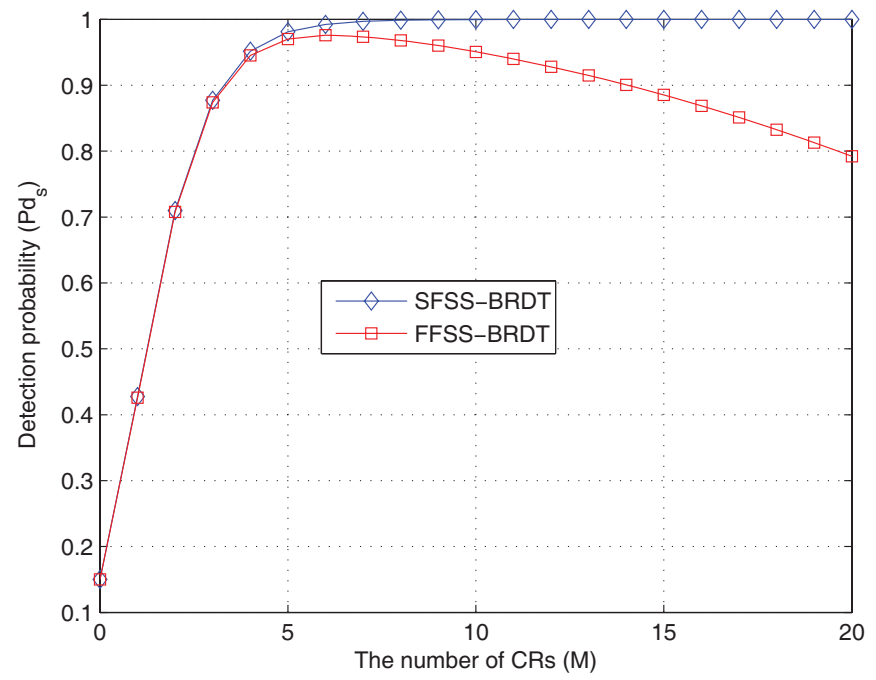

Fig. 4. The detection probability versus the number of cognitive relays of the SFSS-BRDT and the FFSS-BRDT schemes for different $B_{c} T_{c}$ values with $\mathrm{P}_{\mathrm{a}}=0.6, \mathrm{Pf}_{\mathrm{s}}=10^{-3}, \gamma_{p}=20 \mathrm{~dB}, \alpha=0.5, B_{c} T_{c}=100$, and $\sigma_{p s}^{2}=\sigma_{p i}^{2}=\sigma_{i s}^{2}=1$.

All cases in Fig. 4 clearly show that, when the number of CRs is smaller than a critical value, the detection probabilities of the SFSS-BRDT are nearly identical to that of the FFSSBRDT scheme. One can also observe from Fig. 4 that, as the number of cognitive relays continues increasing after the critical value, the detection probability of the FFSSBRDT scheme starts to degrade. This is due to the fact that, with an increased number of CRs and a limited common control channel resources, the channel outage events occurred during the transmission of initial sensing results become more frequent, leading to more initial sensing results received at $\mathrm{CS}$ in error. It then becomes the dominant factor adversely affecting the FFSS-BRDT's sensing performance, since this scheme can not recognize if the received initial sensing result at CS from a CR is in error or not. However, the SFSS-BRDT scheme is able to identify and discard such errors by CRC checking, and thus is not affected by this adverse factor.

Fig. 5 shows the spectrum hole utilization efficiency versus the secondary SNR $\left(\gamma_{s}\right)$ of the non-cooperation, SFSS-BRDT and FFSS-BRDT schemes with a false alarm probability constraint $\operatorname{Pf}_{s}=10^{-3}$, where the time-bandwidth products of the licensed channel and common control channel are $B T=500$ and $B_{c} T_{c}=100$, respectively. This considers that the cognitive radio is typically designed to reuse the licensed spectrum with very limited dedicated channel resources. We also provide the simulated spectrum hole utilization efficiency results by using a typical link-level simulation approach. As observed from Fig. 5, in low SNR regions, both the SFSS-BRDT and FFSS-BRDT schemes perform worse than the non-cooperation scheme in terms of the spectrum hole utilization efficiency. This is because that a half-duplex relaying mode is adopted, which would degrade the system performance due to the inefficient duplexing method. However, in higher SNR regions, the relay benefits achieved overtake the performance cost due to the half-duplexing constraint and thus the SFSS-BRDT and FFSS-BRDT schemes outperform the non-cooperation. Also, it can be seen from Fig. 5 that the spectrum hole utilization

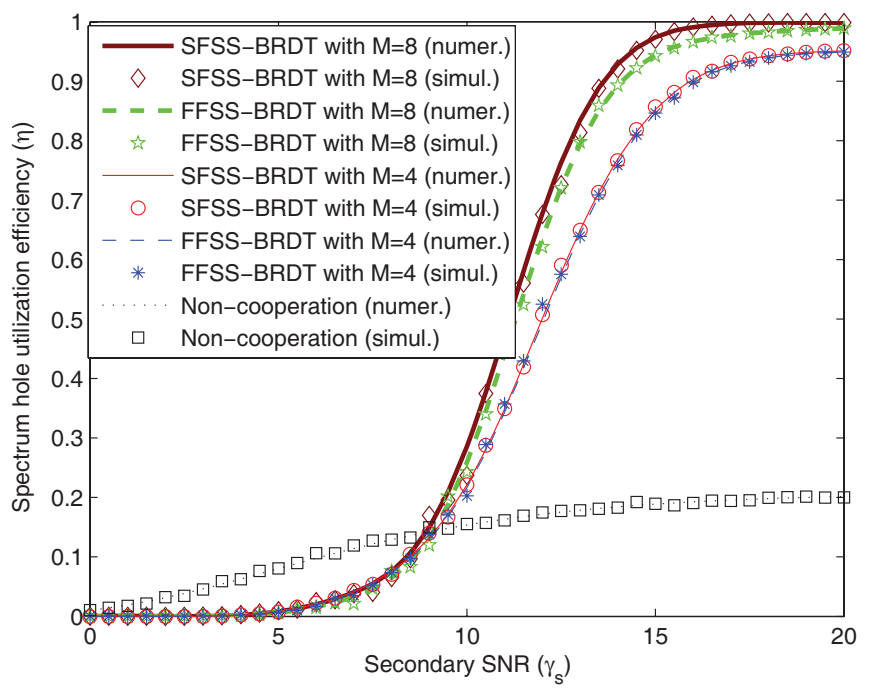

Fig. 5. Spectrum hole utilization efficiency versus the secondary SNR $\gamma_{s}$ of the non-cooperation, the SFSS-BRDT and the FFSS-BRDT schemes for different number of cognitive relays $M$ with $\mathrm{P}_{\mathrm{a}}=0.6, \mathrm{Pf}_{s}=10^{-3}, \gamma_{p}=$ $20 \mathrm{~dB}, R=1 \mathrm{bit} / \mathrm{s} / \mathrm{Hz}, \alpha=0.5, B T=500, B_{c} T_{c}=100$, and $\sigma_{p s}^{2}=$ $\sigma_{p i}^{2}=\sigma_{p d}^{2}=\sigma_{s i}^{2}=\sigma_{s d}^{2}=\sigma_{i s}^{2}=\sigma_{i d}^{2}=1$.

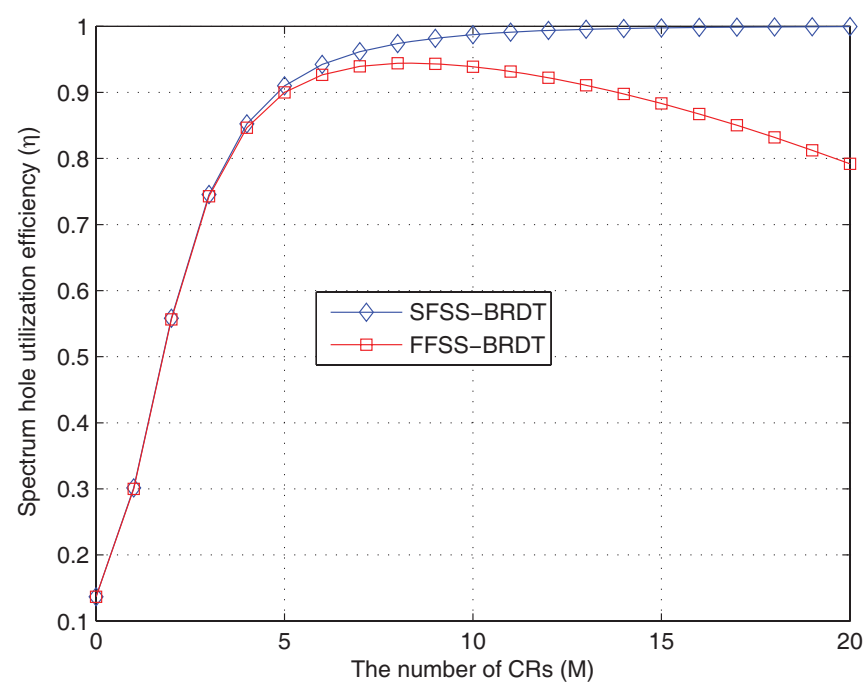

Fig. 6. Spectrum hole utilization efficiency versus the number of cognitive relays $M$ of the SFSS-BRDT and the FFSS-BRDT schemes with $\mathrm{P}_{\mathrm{a}}=0.6$, $\mathrm{Pf}_{s}=10^{-3}, \gamma_{p}=20 \mathrm{~dB}, \gamma_{s}=15 \mathrm{~dB}, R=1 \mathrm{bit} / \mathrm{s} / \mathrm{Hz}, \alpha=0.5, B T=$ $500, B_{c} T_{c}=100$, and $\sigma_{p s}^{2}=\sigma_{p i}^{2}=\sigma_{p d}^{2}=\sigma_{s i}^{2}=\sigma_{s d}^{2}=\sigma_{i s}^{2}=\sigma_{i d}^{2}=1$.

efficiency of the SFSS-BRDT scheme is higher than that of the FFSS-BRDT scheme and, moreover, the performance gap between the two schemes enlarges as the number of cognitive relays increases from $M=4$ to 8 . In addition, the simulations match the analytic results very well, showing the accuracy of the derived closed-form expressions of the spectrum hole utilization efficiency.

In Fig. 6, we illustrate the spectrum hole utilization efficiency of the SFSS-BRDT and FFSS-BRDT schemes versus the number of CRs with a false alarm probability constraint $\mathrm{Pf}_{s}=10^{-3}$. One can see from Fig. 6 that, when the number of cognitive relays is below a certain value, the spectrum hole utilization efficiency of the SFSS-BRDT scheme is almost the same as that of the FFSS-BRDT scheme. However, as 


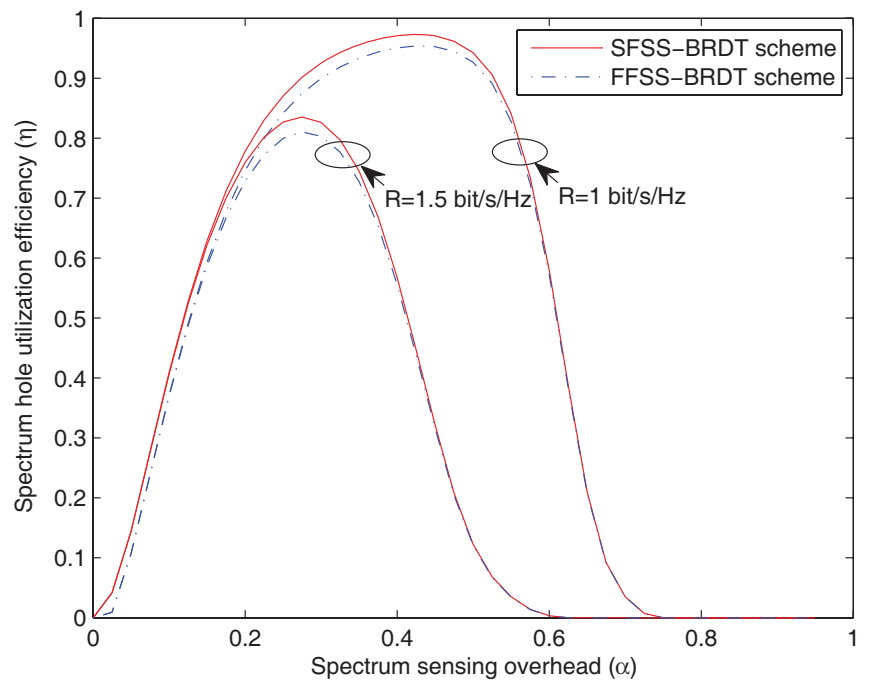

Fig. 7. Spectrum hole utilization efficiency versus the spectrum sensing overhead $\alpha$ of the SFSS-BRDT and the FFSS-BRDT schemes for different data rates $R$ with $\mathrm{P}_{\mathrm{a}}=0.6, \mathrm{Pf}_{s}=10^{-3}, \gamma_{p}=20 \mathrm{~dB}, \gamma_{s}=15 \mathrm{~dB}$, $B T=500, B_{c} T_{c}=100, M=4$, and $\sigma_{p s}^{2}=\sigma_{p i}^{2}=\sigma_{p d}^{2}=\sigma_{s i}^{2}=\sigma_{s d}^{2}=$ $\sigma_{i s}^{2}=\sigma_{i d}^{2}=1$.

the number of CRs continues increasing after a critical value, the FFSS-BRDT scheme's performance begins to degrade and performs noticeably worse than the SFSS-BRDT scheme. This is because that, with an increased number of CRs, the channel outages occurred from CRs to CS will eventually be significant and result in many initial sensing results received at CS in error. Since the FFSS-BRDT scheme can not recognize and discard these errors, it causes the spectrum hole detection failure and in turn affects adversely the spectrum hole utilization efficiency. In contrast, the SFSS-BRDT scheme is able to identify whether an initial sensing result received at CS from a CR is correct or not by the CRC checking, whose spectrum hole utilization efficiency is thus not degraded even with a large number of cognitive relays.

Fig. 7 shows the spectrum hole utilization efficiency versus the spectrum sensing overhead of the SFSS-BRDT and the FFSS-BRDT schemes for different data rates, where the two curve pairs plotted correspond to $R=1 \mathrm{bit} / \mathrm{s} / \mathrm{Hz}$ and $R=$ $1.5 \mathrm{bit} / \mathrm{s} / \mathrm{Hz}$, respectively. As shown in Fig. 7, there always exists an optimal spectrum sensing overhead to maximize the spectrum hole utilization efficiency for both the SFSS-BRDT and the FFSS-BRDT schemes. Therefore, a joint analysis of the two phases (i.e., the spectrum sensing and the data transmission phases) in terms of the spectrum hole utilization efficiency is essential to optimize the overall performance of cognitive transmissions. Fig. 7 also demonstrates that, no matter which scheme (SFSS-BRDT or FFSS-BRDT) is used, the optimal spectrum sensing overhead corresponding to $R=1.5 \mathrm{bit} / \mathrm{s} / \mathrm{Hz}$ is smaller than that for $R=1 \mathrm{bit} / \mathrm{s} / \mathrm{Hz}$. This is due to the fact that as the data rate $R$ increases, the data transmission phase should be allocated a relatively longer time duration, which results in less time available for the spectrum sensing phase. In addition, it is shown from Fig. 7 that the spectrum hole utilization efficiency of the SFSSBRDT scheme is always higher than that of the FFSS-BRDT scheme.

\section{CONCLUSION}

In this paper, we have investigated two cognitive transmission schemes for multiple-relay cognitive radio networks, i.e., the selective fusion spectrum sensing and best relay data transmission scheme and the fixed fusion spectrum sensing and best relay data transmission scheme. We have derived closed-form expressions of the spectrum hole utilization efficiency for the two schemes over Rayleigh fading channels. Numerical results have shown that the SFSS-BRDT scheme outperforms the FFSS-BRDT scheme in terms of the spectrum hole utilization efficiency. Moreover, as the number of CRs increases, the spectrum hole utilization efficiency of the FFSSBRDT scheme improves initially and then begins to degrade. In contrast, the SFSS-BRDT's performance always improves with an increase of the number of CRs, which further verifies its advantage over the FFSS-BRDT scheme. In addition, it has been shown that a maximum spectrum hole utilization efficiency is achieved through an optimization of the spectrum sensing overhead.

\section{APPENDiX A}

PROOF OF EQ. (28)

We can rewrite $\operatorname{Pr}\left\{\max _{i \in D_{m}}\left|h_{i d}(k)\right|^{2}<2 \Delta-\left|h_{s d}(k)\right|^{2}+\right.$ $\left.2\left|h_{p d}(k)\right|^{2} \gamma_{p} \Delta\right\}$ as $\operatorname{Pr}\left\{\max _{i \in D}\left|h_{i d}(k)\right|^{2}<2 \Delta-x\right\}$, where $x=\left|h_{s d}(k)\right|^{2}-2\left|h_{p d}(k)\right|^{2} \gamma_{p} \Delta$. Notice that RVs $\left|h_{s d}(k)\right|^{2}$ and $\left|h_{p d}(k)\right|^{2}$ follow exponential distribution with parameters $1 / \sigma_{s d}^{2}$ and $1 / \sigma_{p d}^{2}$, respectively. Hence, the probability density function of RV $x$ can be given by

$$
f(x)= \begin{cases}\frac{1}{\sigma_{s d}^{2}+2 \sigma_{p d}^{2} \gamma_{p} \Delta} \exp \left(-\frac{x}{\sigma_{s d}^{2}}\right), & x \geq 0 \\ \frac{1}{2 \sigma_{p d}^{2} \gamma_{p} \Delta+\sigma_{s d}^{2}} \exp \left(\frac{x}{2 \sigma_{p d}^{2} \gamma_{p} \Delta}\right), & \text { otherwise }\end{cases}
$$

Thus, we obtain

$$
\begin{aligned}
& \operatorname{Pr}\left\{\max _{i \in D_{m}}\left|h_{i d}(k)\right|^{2}<2 \Delta-x\right\} \\
& =\int_{-\infty}^{\Lambda} \prod_{i \in D_{m}}\left[1-\exp \left(-\frac{2 \Delta-x}{\sigma_{i d}^{2}}\right)\right] f(x) d x \\
& =\mathrm{A}_{m}+\mathrm{B}_{m}
\end{aligned}
$$

where the terms $\mathrm{A}_{m}$ and $\mathrm{B}_{m}$ are given by

$$
\mathrm{A}_{m}=\int_{-\infty}^{0} \frac{\Psi}{2 \sigma_{p d}^{2} \gamma_{p} \Delta+\sigma_{s d}^{2}} \exp \left(\frac{x}{2 \sigma_{p d}^{2} \gamma_{p} \Delta}\right) d x
$$

and

$$
\mathrm{B}_{m}=\int_{0}^{\Lambda} \frac{\Psi}{\sigma_{s d}^{2}+2 \sigma_{p d}^{2} \gamma_{p} \Delta} \exp \left(-\frac{x}{\sigma_{s d}^{2}}\right) d x
$$

wherein the parameter $\Psi$ is given by

$$
\Psi=1+\sum_{n=1}^{2^{\left|D_{m}\right|}-1}(-1)^{\left|S_{m}(n)\right|} \exp \left(-\sum_{i \in S_{m}(n)} \frac{2 \Delta-x}{\sigma_{i d}^{2}}\right)
$$

where $S_{m}(n)$ is the $n$-th non-empty subcollection of the elements of $D_{m}$ and $\left|S_{m}(n)\right|$ is the number of the elements in $S_{m}(n)$. In obtaining Eq. (A.5), we have used binomial 
expansion formula. Substituting $\Psi$ from Eq. (A.5) into Eq. (A.3) yields

$$
\mathrm{A}_{m}=\Gamma+\sum_{n=1}^{2^{\left|D_{m}\right|}-1} \frac{(-1)^{\left|S_{m}(n)\right|} \Gamma}{1+\sum_{i \in S_{m}(n)} \frac{2 \sigma_{p d}^{2} \gamma_{p} \Delta}{\sigma_{i d}^{2}}} \exp \left(-\sum_{i \in S_{m}(n)} \frac{2 \Delta}{\sigma_{i d}^{2}}\right)
$$

where $\Gamma=\frac{2 \sigma_{p d}^{2} \gamma_{p} \Delta}{2 \sigma_{p d}^{2} \gamma_{p} \Delta+\sigma_{s d}^{2}}$. Similarly, substituting $\Psi$ from Eq. (A.5) into Eq. (A.4), we are able to obtain

$$
\begin{aligned}
\mathrm{B}_{m}= & (1-\Gamma)\left\{1-\exp \left(-\frac{2 \Delta}{\sigma_{s d}^{2}}\right)\right. \\
& \left.+\sum_{n=1}^{2^{\left|D_{m}\right|}-1}(-1)^{\left|S_{m}(n)\right|} \phi\left[\sigma_{s d}^{2}, \sigma_{i d}^{2}, S_{m}(n)\right]\right\}
\end{aligned}
$$

where $\phi\left[\sigma_{s d}^{2}, \sigma_{i d}^{2}, S_{m}(n)\right]$ is given by

$\phi\left[\sigma_{s d}^{2}, \sigma_{i d}^{2}, S_{m}(n)\right]=\left\{\begin{array}{l}\frac{2 \Delta}{\sigma_{s d}^{2}} \exp \left(-\frac{2 \Delta}{\sigma_{s d}^{2}}\right), \sum_{i \in S_{m}(n)} \frac{1}{\sigma_{i d}^{2}}=\frac{1}{\sigma_{s d}^{2}} \\ \frac{\exp \left(-\sum_{i \in S_{m}(n)} \frac{2 \Delta}{\sigma_{i d}^{2}}\right)-\exp \left(-\frac{2 \Delta}{\sigma_{s d}^{2}}\right)}{1-\sum_{i \in S_{m}(n)} \frac{\sigma_{s d}^{2}}{\sigma_{i d}^{2}}},\end{array}\right.$ others

wherein $\Delta=\left[2^{2 R /(1-\alpha)}-1\right] / \gamma_{s}$.

\section{REFERENCES}

[1] J. Mitola and G. Q. Maguire, "Cognitive radio: making software radios more personal," IEEE Personal Commun., vol. 6, pp. 13-18, 1999.

[2] IEEE 802.22 Working Group, "IEEE P802.22/D1.0 draft standard for wireless regional area networks part 22: cognitive wireless RAN medium access control (MAC) and physical layer (PHY) specifications: policies and procedures for operation in the TV bands," Apr. 2008.

[3] J. Ma, Y. G. Li, and B. H. Juang, "Signal processing in cognitive radio," Proc. IEEE, vol. 97, no. 5, pp. 805-8023, 2009.

[4] Y.-C. Liang, Y. Zeng, E. Peh, and A. T. Hoang, "Sensing-throughput tradeoff for cognitive radio networks," IEEE Trans. Wireless Commun., vol. 7, no. 4, pp. 1326-1337, Apr. 2008.

[5] Y. Zou, Y.-D. Yao, and B. Zheng, "Outage probability analysis of cognitive transmissions: the impact of spectrum sensing overhead," IEEE Trans. Wireless Commun., vol. 9, no. 8, pp. 2676-2688, Aug. 2010.

[6] V. I. Kostylev, "Energy detection of a signal with random amptitude," in Proc. IEEE Int. Conf. Commun., 2002.

[7] A. Fehske, J. D. Gaeddert, and J. H. Reed, "A new approach to signal classification using spectral correlation and neural networks," in Proc. IEEE DYSPAN 2005, Nov. 2005, pp. 144-150.

[8] P. D. Sutton, K. E. Nolan, and L. E. Doyle, "Cyclostationary signatures in practical cognitive radio applications," IEEE J. Sel. Areas Commun., vol. 26, no. 1, pp. 13-24, 2008 .

[9] A. Ghasemi and E. S. Sousa, "Collaborative spectrum sensing for opportunistic access in fading environment," in Proc. IEEE DySPAN 2005, pp. 131-136.

[10] G. Ganesan and Y. G. Li, "Cooperative spectrum sensing in cognitive radio-part I: two user networks," IEEE Trans. Wireless Commun., vol. 6, no. 6, pp. 2204-2213, 2007.

[11] J. Ma, G. Zhao, and Y. Li, "Soft combination and detection for cooperative spectrum sensing in cognitive radio networks," IEEE Trans. Wireless Commun., vol. 7, no. 11, pp. 4502-4507, Nov. 2008.

[12] R. W. Pabst and B. H. Shultz, et al., "Relay-based deployment concepts for wireless and mobile broadband radio," IEEE Commun. Mag., vol. 42, no. 9, pp. 80-89, Sep. 2004.

[13] A. J. Paulraj, D. A. Gore, R. U. Nabar, and H. Bolcskei, "An overview of MIMO communications-a key to Gigabit wireless," Proc. IEEE, vol. 92, no. 2, pp. 198-218, Feb. 2004.

[14] J. N. Laneman, D. N. C. Tse, and G. W. Wornell, "Cooperative diversity in wireless networks: efficient protocols and outage behavior," IEEE Trans. Inf. Theory, vol. 50, no. 12, pp. 3062-3080, 2004.
[15] A. Sendonaris, E. Erkip, and B. Aazhang, "User cooperation diversitypart I: system description," IEEE Trans. commun., vol. 51, no. 11, pp. 1927-1938, 2003.

[16] T. E. Hunter, S. Sanayei, and A. Nosratinia, "Outage analysis of coded cooperation," IEEE Trans. Inf. Theory, vol. 52, no. 2, pp. 375-391, 2006.

[17] Y. Zou, B. Zheng, and J. Zhu, "Outage analysis of opportunistic cooperation over Rayleigh fading channels," IEEE Trans. Wireless Commun., vol. 8, no. 6, pp. 3077-3385, June 2009.

[18] Y. Zou, B. Zheng, and W.-P. Zhu, "An opportunistic cooperation scheme and its BER analysis," IEEE Trans. Wireless Commun., vol. 8, no. 9, pp. 4492-4497, Sep. 2009.

[19] A. Bletsas, H. Shin, M. Z. Win, and A. Lippman, "A simple cooperative diversity method based on network path selection," IEEE J. Sel. Areas Commun., vol. 24, no. 3, pp. 659-672, Mar. 2006.

[20] E. Beres and R. S. Adve, "Selection cooperation in multi-source cooperative networks," IEEE Trans. Wireless Commun., vol. 7, no. 1, pp. 118-127, 2008.

[21] S. Lkki and M. H. Ahmed, "Performance of multiple-relay cooperative diversity systems with best relay selection over Rayleigh fading channels," EURASIP J. Advances in Signal Process., article ID 580368.

[22] Z. Tian and G. B. Giannakis, "A wavelet approach to wideband spectrum sensing for cognitive radios," in Proc. IEEE CROWNCOM 2006, pp. 1-5.

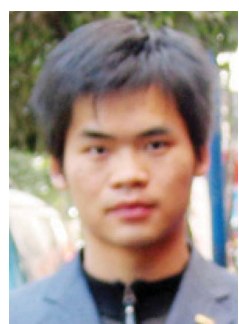

Yulong Zou received his B.Eng. degree (with the highest honor) in Information Engineering from Nanjing University of Posts and Telecommunications (NUPT), Nanjing China, in 2006. He is currently working toward his Ph.D. degrees, respectively, at the Institute of Signal Processing and Transmission of NUPT, Nanjing China, and the Electrical and Computer Engineering Department of Stevens Institute of Technology (SIT), NJ USA. His research interests span the broad area of scalable wireless communication and networking, with emphasis on cooperative relay technology. Recently, he focuses on the investigation of cooperative relay techniques in cognitive radio networks.

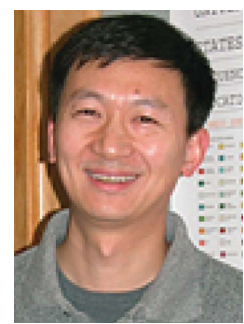

Yu-Dong Yao (S'88-M'88-SM'94) has been with Stevens Institute of Technology, Hoboken, New Jersey, since 2000 and is currently a professor and department director of electrical and computer engineering. He is also a director of Stevens' Wireless Information Systems Engineering Laboratory (WISELAB). Previously, from 1989 and 1990, he was at Carleton University, Ottawa, Canada, as a Research Associate working on mobile radio communications. From 1990 to 1994, he was with Spar Aerospace Ltd., Montreal, Canada, where he was involved in research on satellite communications. From 1994 to 2000, he was with Qualcomm Inc., San Diego, CA, where he participated in research and development in wireless code-division multiple-access (CDMA) systems.

He holds one Chinese patent and twelve U.S. patents. His research interests include wireless communications and networks, spread spectrum and CDMA, antenna arrays and beamforming, cognitive and software defined radio (CSDR), and digital signal processing for wireless systems. Dr. Yao was an Associate Editor of IEEE Communications Letters and IEEE TRANSACTIONS ON VEHICULAR TECHNOLOGY, and an Editor for IEEE TRANSACTIONS ON WiRELESS COMMUNICATIONS. He received the B.Eng. and M.Eng. degrees from Nanjing University of Posts and Telecommunications, Nanjing, China, in 1982 and 1985, respectively, and the Ph.D. degree from Southeast University, Nanjing, China, in 1988, all in electrical engineering.

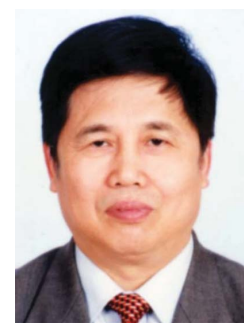

Baoyu Zheng received B.S. and M.S. degrees from the Department of Circuit and Signal System, NUPT, in 1969 and 1981, respectively. Since then, he has been engaged in teaching and researching at Signal and Information Processing. He is a full professor and doctoral advisor at NUPT. His research interests span the broad area of the intelligent signal processing, wireless network and signal processing for modern communication, and the quantum signal processing. 